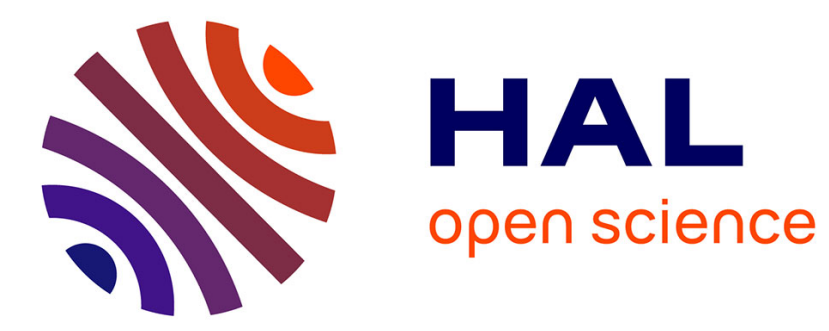

\title{
La loi Dutreil-Jacob, nouvel épisode de l'encadrement juridique des négociations tarifaires entre la grande distribution et le monde industriel
}

Odile Chanut

\section{- To cite this version:}

Odile Chanut. La loi Dutreil-Jacob, nouvel épisode de l'encadrement juridique des négociations tarifaires entre la grande distribution et le monde industriel . 2007. hal-01761590

\author{
HAL Id: hal-01761590 \\ https://hal.science/hal-01761590
}

Preprint submitted on 11 Apr 2018

HAL is a multi-disciplinary open access archive for the deposit and dissemination of scientific research documents, whether they are published or not. The documents may come from teaching and research institutions in France or abroad, or from public or private research centers.
L'archive ouverte pluridisciplinaire HAL, est destinée au dépôt et à la diffusion de documents scientifiques de niveau recherche, publiés ou non, émanant des établissements d'enseignement et de recherche français ou étrangers, des laboratoires publics ou privés. 


\section{La loi Dutreil-Jacob, nouvel épisode de l'encadrement juridique des négociations tarifaires entre la grande distribution et le monde industriel}

\section{Odile Chanut, Université de Savoie}

La loi Galland avait 10 ans. Critiquée pour les effets pervers qu'elle a entraînés, inflation et hausse importante des marges arrière, elle a été réformée par la loi du 2 août 2005 en faveur des petites et moyennes entreprises, dite loi Dutreil-Jacob. La circulaire du 8 décembre 2005 relative aux relations commerciales, qui précise la loi, est parue au JO du 30 décembre 2005. Le nouveau dispositif affiche un objectif, la baisse des prix des produits de grande marque, et deux moyens, le basculement des marges arrière vers les marges avant et l'encadrement strict, assorti de sanctions pénales lourdes, des facturations de services aux industriels par les distributeurs. L'article se propose de présenter, de manière synthétique, la nouvelle réglementation relative aux conditions tarifaires des relations commerciales entre industriels et distributeurs. Une analyse critique du nouveau texte est ensuite proposée. Deux aspects sont analysés : la possibilité d'une réelle baisse des prix, qui pourrait être limitée ; et l'impact du dispositif sur les relations des acteurs de la chaîne productiondistribution. La thèse défendue est que le nouveau dispositif crée de l'effervescence dans les états majors des acteurs mais n'est pas de nature à modifier les rapports de force installés.

\section{Introduction}

Sans le référencement dans les linéaires de la grande distribution alimentaire, pas d'accès aux consommateurs. C'est pourquoi les industriels se voient imposer des conditions tarifaires drastiques par des distributeurs, d'autant plus forts qu'ils constituent en France un oligopsone (voir encadré 1). Si la grande distribution, alliée de taille dans la lutte contre l'inflation, a été plutôt ménagée par les pouvoirs publics jusqu'en 1996, dans un contexte d'inflation désormais maîtrisée, le législateur est intervenu depuis avec pour objectif affiché de rétablir l'équilibre dans le pouvoir de négociation des acteurs du canal de la grande distribution alimentaire. Pas moins de sept textes de droit ont été adoptés depuis dix ans, apportant des limites aux principes de liberté contractuelle, de libre jeu de la concurrence ou encore de celui de liberté des prix posée par l'ordonnance du $1^{\text {er }}$ décembre 1986 : quatre lois, deux circulaires, un accord interprofessionnel, ce qui représente un texte en moyenne tous les 18 mois. La remarque de Villain (13) conserve sa pertinence : «La législation française sur les rapports verticaux producteurs-distributeurs est, de loin, la plus développée. Son pointillisme, c'est-à- 
dire son intrusion dans le détail de la transaction bilatérale commerciale, est unique ». Cette opinion a été à nouveau illustrée par l'adoption, en août 2005 d'une loi en faveur des petites et moyennes entreprises, dont le titre VI, qui porte sur la modernisation des relations commerciales, vient réformer la loi Galland du 1er juillet 1996. La circulaire du 8 décembre 2005 précise la loi (7).

\section{Encadré 1 ici}

Nous rappelons brièvement les principaux effets «pervers » de la loi Galland qui ont motivé la réforme, puis nous présentons les innovations juridiques que cette dernière introduit concernant les négociations tarifaires. Une analyse critique du nouveau dispositif, au regard de l'objectif affiché, la baisse des prix, et de l'impact de la réforme sur les relations des acteurs du canal est ensuite proposée. La thèse défendue est que le nouveau dispositif crée de l'effervescence dans les états majors des acteurs mais n'est pas de nature à modifier les rapports de force installés. Ni véritablement à imposer une baisse des prix significative.

\section{Les principaux effets « pervers » du dispositif avant la réforme de 2005}

Deux dispositions de la loi Galland doivent être rappelées : les règles de facturation et l'interdiction de la discrimination tarifaire. D'une part, la loi a modifié les règles de facturation, pour accroître la transparence tarifaire et rendre effective l'interdiction de revente à perte. Le délit de revente à perte existe en droit français depuis la loi de finances du 2 juillet 1963. Toutefois, l'interdiction était contournée grâce à l'art de la «facturologie », ou science de la manipulation des factures (11), qui consistait à imputer, sur certaines factures, des remises étrangères à l'achat considéré. Depuis la loi Galland, seules peuvent apparaître, sur une facture d'achat de produits, les remises non conditionnelles liées directement à l'opération. Toute autre forme de remise est exclue, en particulier les budgets réclamés par les distributeurs pour des services proposés au titre de la coopération commerciale, tels que la mise en avant des produits, l'attribution de têtes de gondole, la publicité sur le lieu de vente, les catalogues publi-promotionnels etc.. Auparavant déduits des factures sous formes de remises, rabais, ristournes, les fameux «RRR», ces services doivent désormais faire l'objet de facturation séparée, autonome, d'où le nom de «marges arrière ». D'autre part, la loi incitait les industriels à instaurer des conditions générales de vente, base de la négociation, et rendait plus aisée la preuve de la discrimination tarifaire, sanctionnée depuis 1958. Les 
industriels doivent proposer des conditions identiques aux distributeurs présentant les mêmes profils d'acheteurs. Ces nouvelles règles ont eu deux effets pervers : l'inflation et la hausse très importante des marges arrière.

\section{L'inflation}

Le seuil de revente à perte, qui constitue un plancher pour le prix de revente, était désormais contrôlé par les industriels. Les conditions générales de vente étant les mêmes pour toutes les centrales d'achat, c'était la fin de la guerre des prix entre distributeurs (concurrence intramarque), et le rapprochement des prix entre les formats des points de vente (hypermarchés, supermarchés et supérettes). La conséquence a été l'harmonisation, à la hausse, des prix de vente aux consommateurs, grands «perdants » de la loi, et notamment des prix des grandes marques. Une étude a mise en évidence des augmentations moyennes de prix, entre 2000 et 2003 , de $11,2 \%$ pour les grandes marques, contre $4,3 \%$ pour les marques de distributeur et $4,1 \%$ pour les produits premiers prix (10).

\section{Le niveau particulièrement élevé des marges arrière}

La facturation autonome des services aurait du permettre d'accroître la transparence tarifaire des services de coopération commerciale. Mais le mécanisme a été détourné de son objet. Les distributeurs ont choisi de ne plus se rémunérer par une marge commerciale sur les produits. Ils ont fixé les prix de vente consommateur au niveau du seuil de revente à perte, et ont gonflé la facturation des marges arrière pour maintenir leur niveau de rémunération. Les marges arrière ont alors connu des hausses très importantes, et ont représenté parfois des sommes allant jusqu'à $60 \%$ du CA net facturé. A titre de comparaison, le niveau moyen de la coopération commerciale, à l'étranger serait de $20 \%$, comparable à celui que l'on pouvait observer en France avant l'adoption de la loi Galland (10).

Surtout, la loi Galland n'a pas été de nature à modifier le rapport de force en faveur des distributeurs observé depuis une vingtaine d'années. Le bilan est toutefois contrasté selon les acteurs du canal.

\section{Un bilan contrasté selon les acteurs du canal}

Les consommateurs ont été perdants, subissant des hausses de prix pour les grandes marques supérieures de $80 \%$ à la hausse générale des prix (10).

Les distributeurs ont maintenu leurs marges et leur pouvoir de négociation. Une redistribution des parts de marché s'est toutefois opérée entre les différents formats, au profit des magasins 
de proximité, et au profit du hard-discount, vers lequel se sont tournés les consommateurs soucieux de maintenir leur pouvoir d'achat. Les hypermarchés ont souffert, perdant leur positionnement prix historique.

Les groupes industriels multinationaux, capables d'opposer à la puissance d'achat des distributeurs une puissance marketing et de communication, ont également été bénéficiaires. Retrouvant la maîtrise des prix par le biais du jeu du seuil de revente à perte (le SRP, voir l'encadré 6), ils ont consolidé leurs marges industrielles.

Le bilan pour les PME est plus mitigé. Les marques propres des PME ont parfois du mal à se maintenir sur les linéaires, devenus rares sous l'effet de la loi Raffarin. La dépendance des PME s'est accrue. Certes les 5200 PME françaises fournisseurs de la grande distribution bénéficient du développement des marques de distributeurs (MDD), qu'elles fabriquent en grande majorité, et qui ne donnent pas lieu à marges arrière. Mais les distributeurs contrôlent la chaîne de valeur des produits vendus sous MDD, et font régulièrement pression pour obtenir des réductions de prix. Le résultat est une baisse effective des prix des MDD de 2,6\% en 2005, alors que sur la même période les prix des grandes marques augmentaient de 0,4\% (10). Il faut toutefois modérer ce propos en distinguant les MDD premier prix, dont l'offre a augmenté pour faire face au hard-discount, expliquant la tendance des prix moyens, des MDD «me too » qui recherchent un bon rapport qualité-prix par rapport à la marque de référence, et des MDD à forte valeur ajoutée, innovantes ou thématiques, telles que «Reflets de France » ou la gamme bio de CARREFOUR. Le risque pour les PME est toutefois de se voir cantonner dans leur rôle de «fournisseurs de MDD ». Le tableau 1 détaille les intérêts divergents des acteurs du canal de la grande distribution, les menaces auxquelles ils sont exposés, les réponses possibles, et les positions de chacun face à la loi Galland.

Tableau 1 ici

La réforme de la loi Galland, annoncée par la circulaire dite «Dutreil I» et l'accord interprofessionnel dit «Sarkozy», avait pour objectif de corriger ses effets pervers et de favoriser les conditions de la baisse des prix des produits de grande marque.

\section{La nouvelle réglementation des pratiques commerciales}


Le titre VI de la loi $n^{\circ} 2005-882$ du 5 août 2005 relatif à la modernisation des relations commerciales, précisé par la circulaire du 8 décembre 2005 (7), comporte plusieurs volets ayant pour objectif de protéger les PME contre des pratiques jugées abusives, qui les fragilisent. Citons par exemple la limitation des accords de gamme qui peuvent conduire à évincer les marques propres des PME des linéaires, du fait de la rareté de ces derniers. Ces dispositions constituent des adaptations de la réglementation aux pratiques commerciales en perpétuelle évolution. Nous ne les détaillons pas toutes dans cet article centré sur les nouvelles dispositions concernant les négociations tarifaires entre industriels et grands distributeurs.

\section{Le plafonnement des marges arrière pour favoriser la baisse des prix de vente aux consommateurs}

L'objectif principal des nouveaux textes est de favoriser la baisse des prix de vente aux consommateurs. Cela passe par une nouvelle définition du Seuil de Revente à Perte (SRP) et par le plafonnement des marges arrière à $20 \%$ en 2006 et à $15 \%$ en 2007.

\section{Nouvelle définition du seuil de revente à perte (SRP)}

Le SRP est le prix plancher, en-dessous duquel le distributeur ne peut pas revendre les produits, sauf à enfreindre l'interdiction de revente à perte et à s'exposer à des sanctions pénales lourdes. Il est égal au prix d'achat effectif dont la définition a évolué dans le temps, en fonction des objectifs du législateur pour infléchir les pratiques des professionnels.

Avant la loi Galland, le SRP était le prix d'achat unitaire moins «les remises, rabais et ristournes dont le principe était acquis et le montant chiffrable au jour de la vente » (rédaction de l'article L 441-3 du code de commerce antérieure à 1996). Ces «RRR » incluaient des éléments très disparates, parfois sans lien avec l'opération d'achat de marchandises : les remises quantitatives ou qualitatives, mais aussi des réductions pour financer la mise à disposition de tête de gondole, ou les prospectus distributeur. Fréquente était la pratique dite de la «cagnotte » qui consistait à imputer sur une facture, celle du mois de septembre par exemple, les remises cumulées depuis le début de l'année et rétroactives car soumises à une condition de volume atteinte en septembre. Le distributeur pouvait alors proposer en septembre un prix très bas au consommateur, sans enfreindre la réglementation sur la vente à perte. 
La loi Galland avait condamné ces pratiques en modifiant la rédaction de l'article L 441-3 du code de commerce : seules les réductions de prix «acquises à la date de la vente ou de la prestation de service et directement liées à l'opération » pouvaient désormais être déduites du prix d'achat unitaire figurant sur la facture pour le calcul du SRP. La pratique de la cagnotte n'était plus possible, ni la facturation de services non directement liés à l'opération d'achat. Ces derniers devaient faire l'objet de facturation séparée, autonome, dans un contrat de coopération commerciale.

Le dispositif Dutreil-Jacob revoit la définition du SRP pour 2006, en le minorant des marges arrière excédant $20 \%$, avec une réduction plafonnée à $40 \%$ du montant total des marges pratiquée. Ainsi, le SRP Dutreil se situe à un niveau inférieur au SRP Galland et autorise une baisse effective, et significative, du prix de vente au consommateur. La baisse devrait s'accentuer en 2007, avec un SRP encore minoré, les marges arrières étant limitées à $15 \%$. L'étape suivante pourrait-être le «triple net», c'est-à-dire le droit, pour les enseignes, d'utiliser toute la marge arrière, si elles le souhaitent, pour baisser leur prix.

\section{Exemple chiffré}

Le tableau 2 présente les définitions successives du SRP ainsi qu'un exemple chiffré de son calcul selon les différentes définitions, et montre les effets potentiels sur les prix de vente au consommateur. Il reprend l'exemple, développé dans la circulaire du 8 décembre 2005, d'un produit dont le prix achat tarif est de 110, les remises sur factures de 10, et dont l'ensemble des avantages financiers autres (les marges arrière) sont de 35 (soit $35 \%$ du prix unitaire net). Les taxes et prix du transport seront tenus pour négligeables dans l'exemple.

\section{Tableau 2 ici}

Un encadrement strict des contrats de coopération commerciale...et autres contrats

Le nouveau dispositif entend sanctionner les marges arrière qui ne seraient pas justifiées par des services réels rendus par le distributeur à l'industriel. Il entend ainsi lutter contre la tendance des distributeurs à facturer des services fictifs aux industriels pour augmenter leurs profits. Sont visés les budgets facturés aux industriels qui relèvent du métier de distributeur, par exemple la mise en rayon des produits, le merchandising, la publicité sur le lieu de vente, ou encore la facturation de «fausse coopération », qui n'a pas de contenu ou de contrepartie réelle. Les tribunaux ont eu l'occasion de sanctionner la facturation au titre de coopération commerciale de montants ne correspondant à aucun service rendu. 
L'encadré 2 relate la décision du 15 novembre 2005 du tribunal de commerce de Nanterre, qui a condamné la centrale d'achat de LECLERC, Le Galec, à rembourser un peu plus de 23 millions d'euros à 23 industriels du secteur des produits frais, pour avoir établi des « contrats de coopération commerciale à effet rétroactif [...] ne correspondant pas à un service rendu ». Le jugement prévoyait en outre un mécanisme original pour le remboursement des sommes aux industriels : le Trésor Public se chargerait du recouvrement et rembourserait ensuite les sommes aux industriels. Car ces derniers n'oseraient probablement pas les réclamer à LECLERC, qui représente $20 \%$ de leurs chiffre d'affaires potentiels. Le jugement a été infirmé en appel car un vice de procédure était soulevé (Cour d'appel de Versailles, 3 mai 2007). Il n'en reste pas moins exemplaire de la volonté des magistrats de sanctionner sévèrement les services fictifs.

\section{Encadré 2 ici}

Ainsi, une nouvelle grille est créée pour encadrer les allocations de budgets des industriels aux distributeurs, avec des définitions précises pour les quatre catégories créées : les conditions générales de vente, les conditions particulières de vente, les contrats de coopération commerciale, les services distincts. Cette grille est présentée dans le tableau 3. De plus, le formalisme juridique des «négociations de fin d'année » est renforcé, puisque désormais la coopération commerciale devra faire l'objet d'un contrat écrit au plus tard le 15 février pour l'année en cours. Ce peut être un contrat cadre annuel, suivi de plusieurs contrats d'application.

Tableau 3 ici

\section{Les conséquences prévisibles de la nouvelle réglementation}

La nouvelle réglementation pose aux distributeurs à la fois des problèmes pratiques et des questions stratégiques. Nous brossons dans cette troisième partie quelques effets de la réforme, tant pour les distributeurs que pour le monde industriel.

\section{Un risque pénal accru pour les distributeurs}

Dans le nouveau dispositif, le distributeur choisit la base de calcul du SRP, à partir des CGV transmis par les industriels. Il s'expose de ce fait à un risque pénal accru, d'autant que c'est à 
lui désormais d'apporter la preuve de la réalité des services rendus, et facturés aux industriels au titre de la coopération commerciale ou des services distincts, du fait du renversement de la charge de la preuve prévu par la circulaire, dont le mécanisme est expliqué dans l'encadré 3 .

\section{Encadré 3 ici}

Aussi, un certain nombre de problèmes pratiques se posent aux distributeurs : comment fixer sans risque le prix d'une prestation telle que l'attribution d'une tête de gondole, ou un service de collaboration internationale ? Comment va s'opérer le contrôle ? Comment rédiger les accords qui doivent intervenir avant le 15 février chaque année? Que mettre dans les conditions particulières de vente et comment le justifier pour limiter le risque de discrimination? La tentation est grande, pour limiter le risque juridique, de requalifier certains services facturés, par exemple en transférant les budgets correspondant en coûts de référencement justifiés par l'innovation. Les coûts de référencement auraient ainsi exploser dans certaines enseignes.

\section{Une liberté d'action sur les prix retrouvée pour les distributeurs}

La loi Dutreil-Jacob lève en partie le contrôle des prix par les industriels. Elle réintroduit de la liberté d'action sur les prix pour les distributeurs, qui pourront les baisser sans enfreindre l'interdiction de revente à perte. C'est l'objectif affiché de la loi. Elle autorise à nouveau une concurrence sur les prix entre enseignes. Les distributeurs peuvent toutefois hésiter à s'engager dans une "guerre des prix » qui aboutirait à une baisse générale de leurs marges unitaires. L'équation des distributeurs est simple: une baisse des marges unitaires des produits de grande marque devra être compensée. Plusieurs solutions sont théoriquement envisageables : soit des hausses de volumes de vente, soit la création de nouvelles sources de rémunération, soit des baisses de coûts. Nous les envisageons tour à tour.

\section{Hausse peu probable des volumes de vente des GMS « classiques »}

Une hausse des volumes de vente des produits de grande consommation est peu probable. La part de l'alimentaire dans le budget des ménages, en constante diminution, ne représente plus que $14 \%$ contre $28 \%$ en 1960 . Devant la stagnation de leur pouvoir d'achat, les ménages font des arbitrages au profit du logement, du carburant, des supports de communication. Par ailleurs la progression du hard-discount rend difficile le maintien des parts de marché des GMS « classiques » (voir encadré 4$)$. 


\section{Encadré 4 ici}

Reste la possibilité de redistribution de parts de marché entre distributeurs, au profit de ceux qui pourront se distinguer, soit par une politique de prix agressive, mais dangereuse et coûteuse, soit par une offre à forte valeur ajoutée perçue par les clients.

Les relevés de prix un an après l'entrée en vigueur de la loi ne montrent ni guerre des prix, ni baisses drastiques. Plusieurs enseignes communiquent sur une baisse de leurs prix certes et notamment pour les hypermarchés qui ont souffert ces dernières années et cherchent à reconquérir les clients avec un retour au positionnement discount. Mais c'est sur le terrain des promotions que ces distributeurs s'expriment. Les prix permanents, eux n'évoluent guère, en moyenne. Au moins n'augmentent-ils plus au rythme de plusieurs points comme les années précédentes. Ce qui amène certains observateurs à conclure : «Faut-il s'interroger sur l'éventualité d'ententes sur les prix entre les différentes enseignes de la grande distribution et leurs fournisseurs ?»(4). Il est certain qu'une guerre des prix sur les marques nationales pourrait pénaliser tous les distributeurs. Aussi aucune enseigne n'a encore franchi le pas un an après l'entrée en vigueur de la réforme. Un directeur général d'une grande enseigne nous confiait toutefois : «on a une visibilité courte, la situation est dangereuse quand même, il suffit qu'il y en ait un qui allume le pétard, il serait kamikaze, mais enfin on a chacun ses stratégies dans les entreprises...».

La voie de la différenciation par l'offre est plus probable. Les enseignes réduisent le nombre des références pour se centrer sur celles qui représentent les meilleures performances. Elles poursuivent l'augmentation de la place en linéaire des Marques de Distributeurs (MDD), qui dégagent des marges plus importantes pour les distributeurs, parce qu'ils partagent avec les PME qui les fabriquent la marge industrielle. Les MDD représentent, en mai 2006, 29,1\% de l'offre des produits de grande consommation, hors hard-discount, et 25,7\% du CA, en progression de $1 \%$ sur un an (12), contre près de $45 \%$ en Grande Bretagne. Certaines études mettent en évidence le risque de saturation pouvant se traduire par des baisses de fréquentation des magasins, dès que les MDD atteignent $18 \%$ des références en supermarché et $21 \%$ en hypermarché (2). Les distributeurs estiment cependant qu'une marge de progression existe, d'autant que les MDD participent à la définition de la personnalité de l'enseigne, et constituent un terrain de coopération avec les PME valorisant pour les 
distributeurs en recherche de légitimité institutionnelle et d'image d'entreprise citoyenne aux yeux des consommateurs et de l'Etat (9). La voie de la différenciation pourrait aussi être facilitée par l'accès à la publicité télévisée, qui est ouverte aux distributeurs depuis le $1^{\text {er }}$ janvier 2007. La télévision hertzienne pourrait constituer un vecteur appréciable pour nourrir et enrichir l'image d'enseigne, avec des spots institutionnels.

\section{Nouvelles sources de rémunération}

La hausse des marges arrière n'a pas affecté de la même manière l'ensemble des rayons des grandes surfaces alimentaires. Certains rayons connaissent des marges arrière inférieures au plafond de $20 \%$ défini par la nouvelle réglementation, notamment le non-alimentaire. Par exemple, les marges arrière étaient de 15 à $16 \%$ pour le bricolage en 2005. Aussi, les distributeurs pourraient-ils être tentés de reconstituer les marges perdues sur l'alimentaire, en augmentant leurs marges sur d'autres rayons. Le bricolage mais aussi le petit et moyen électroménager, le jouet etc. pourraient en faire les frais.

\section{Baisse des coûts}

Le troisième volet sur lequel peuvent agir les distributeurs pour compenser une baisse des marges unitaires sur les produits de grande marque est la réduction des coûts de structures. Les équipes s'étaient étoffées avec la montée de la puissance de négociation des distributeurs, surtout chez les groupes intégrés. Chez les distributeurs dits «indépendants » (LECLERC, INTERMARCHE, SYSTEME U), le tiers temps que doit chaque adhérent à la centrale d'achat a permis de limiter la taille des structures centrales. Les coûts de structure y seraient inférieurs de 5 points à ceux des structures intégrées (3). De ce fait, une guerre des prix leur serait moins préjudiciable, d'autant qu'ils n'ont pas à se préoccuper de leur cours en bourse. Ils auraient une marge de manœuvre à la baisse des prix supérieure à celle des groupes de distribution intégrés. Cela explique en partie les choix de communication d'une enseigne comme LECLERC, qui a fait de la lutte des prix un «combat de toujours ». Un exemple récent de ces «coups médiatiques» de l'enseigne est le lancement du site «Quiestlemoinscher.com » mis en ligne le 22 mai 2006, suspendu par décision de justice le 31 mai 2006, et relancé, sous une forme différente, le 26 novembre 2006.

\section{Les effets de la réforme pour les industriels}

Du coté des industriels, il semblerait que les premières négociations après l'entrée en vigueur de la loi aient été plutôt mieux vécues que les précédentes. Des questions pratiques se sont 
posées toutefois. Quelle orientation donner aux conditions générales de vente ? Que mettre dans les conditions particulières sans risquer d'être condamnés pour discrimination? Certains industriels craignent de perdre le contrôle des prix consommateurs, du fait de la liberté redonnée aux distributeurs. Et d'avoir à partager la baisse des marges si une guerre des prix entre distributeurs est effective. D'où la tentation de prévoir des hausses des tarifs significatives, pour atténuer l'effet de la loi sur le SRP, et les prix, et pour garder une marge de manœuvre dans les négociations. Le jeu normal des négociations annuelles, qui consistent pour les industriels à annoncer des hausses de tarifs, et pour les distributeurs à annoncer des restrictions des linéaires allouées à la marque, risque de se durcir. Elles seront au moins limitées dans le temps du fait de la date butoir du 15 février fixée par la loi. C'est une innovation de la loi appréciée des industriels qui se voyaient parfois imposer des négociations interminables. L'encadré 5 expose comment les industriels dotés d'un fort pouvoir de négociation, du fait de leur puissance marketing, peuvent atténuer les effets de la loi DutreilJacob par une hausse significative de leurs tarifs. Ainsi, certaines multinationales auraient relevé leur tarif 2006 de 5 à $10 \%$ par rapport à 2005 pour les produits « incontournables » car réclamés par les consommateurs (1). Ces hausses sont parfois justifiées par l'accroissement des coûts de production, notamment de l'énergie et de la main d'œuvre. Mais l'argument n'est pas totalement convaincant quand on constate que ces hausses ne concernent que la France.

\section{Encadré 5 ici}

Quant aux PME, elles vont bénéficier d'une hausse des ventes des MDD qu'elles fabriquent en grande majorité, si la progression de ces dernières se poursuit. Une situation d'autant plus favorable qu'après une phase de développement des MDD premiers prix qui tirait les prix vers le bas, l'heure semble à nouveau être au développement des MDD qualitatives. Mais l'inconnue concernait le sort de leurs marques propres. Certains prédisaient leur éviction des linéaires du fait de la compression des références de marques de fabricants. Au contraire, une étude réalisée par ACNielsen pour le compte de la Fédération des entreprises et entrepreneurs de France, la Feef, montre une progression des marques propres des PME (12). Ces dernières représentent, fin mai 2006, 31,6 \% du CA des produits de grande consommation, en supermarchés et hypermarchés, hors hard-discount, soit une hausse de 0,2\% sur un an et 29,1 $\%$ de l'offre des magasins, avec des progressions sensibles dans certains secteurs tels que les liquides ou le réfrigéré. Les explications avancées de la consolidation de la position des PME sont le durcissement de la réglementation sur les accords de gamme, généralement bénéfiques 
aux grands groupes, mais aussi la réactivité et la souplesse des PME, qui restent des atouts dans les négociations avec les distributeurs.

\section{Conclusion}

La nouvelle réglementation introduit à la fois plus de liberté et plus de responsabilité dans les négociations entre industriels et distributeurs. Elle met fin à «l'encadrement des prix » qui a résulté de la loi Galland. Elle ouvre de nouvelles perspectives de différenciation par les prix pour les distributeurs. Elle offre des outils pour sanctionner les abus des distributeurs à la recherche de marges sans contrepartie réelle.

Est-elle en mesure de favoriser une baisse sensible des prix aux consommateurs des produits de grande marque ? Sur le plan technique, la nouvelle définition du SRP l'autorise. Sur le plan financier, ni les distributeurs, ni les multinationales n'ont intérêt à une guerre des prix. Reste l'action possible des consommateurs, s'ils prenaient conscience de leur pouvoir collectif pour exercer des pressions sur la grande distribution alimentaire. Les armes pourraient être le détour des grandes marques et des GMS «classiques » au profit des MDD et du hard-discount.

Est-elle en mesure de faire évoluer le rapport de force entre industriels et distributeurs ? On peut en douter à court terme, tant que le marché de la grande distribution alimentaire reste «fermé ». Il ne permet guère actuellement l'arrivée de nouveaux distributeurs, étrangers notamment, du fait des barrières à l'entrée créées par les règles restrictives d'urbanisme commercial, durcies par la loi Raffarin du 5 juillet 1996. Cette loi soumet, depuis dix ans, à autorisation administrative toute ouverture ou agrandissement de surfaces de vente supérieures à $300 \mathrm{~m} 2$, contre $1000 \mathrm{~m} 2$ auparavant. Elle pourrait toutefois être remise en cause prochainement: la Commission Européenne, saisie par ALDI, distributeur d'origine allemande qui souhaiterait pouvoir multiplier ses points de vente en France, a en effet mis en demeure la France, le 5 juillet 2006, de modifier cette législation qu'elle juge non conforme aux principes de la liberté d'établissement. Une réforme de la loi Raffarin est depuis annoncée par le gouvernement pour 2007 (6). Nouvelle occasion de remettre à plat l'encadrement juridique de l'activité de distribution... 


\section{Références}

(1) Aubril S. (2006), Les négociations se déroulent à l'arraché, LSA, 1938, 2 février 2006, 2427.

(2) Bitoum O. (2006), Le risque de trop plein de MDD fait débat, LSA, 1954, 25 mai 2006, 24-25.

(3) Canivet G. (2004), Rapport du groupe d'experts constitué sur les Rapports entre industrie et commerce.

(4) Ebran M. (2006), Avis de tempête sur les prix, Que Choisir, 434, février 2006, 50-55.

(5) Gagne F. et Miannay E. (2006), Hard-discount, les low-costs de l'alimentaire, Que Choisir, 440, septembre 2006, 16-23.

(6) Lecompte F. (2006), L'urbanisme commercial sous pression, LSA, 1966, 21 septembre 2006, 50-56.

(7) Loi ${ }^{\circ} 96-588$ du $1^{\text {er }}$ juillet 1996, dite loi Galland, sur la loyauté et l'équilibre des relations commerciales, publiée au JO du 3 juillet 1996 ; Loi $n^{\circ} 2005-882$ du 2 août 2005, dite loi Dutreil-Jacob, en faveur des petites et moyennes entreprises, publiée au JO du 3 août 2005 ; ces lois sont codifiées dans le Code du commerce, Livre quatrième « De la liberté des prix et de la concurrence », titre quatrième «De la transparence, des pratiques restrictives de concurrence et d'autres pratiques prohibées », Chapitre premier «De la transparence », Article L. 441-1 et suivants. Circulaire du 8 décembre 2005 relative aux relations commerciales, parue au JO du 30 décembre 2005.

(8) Malaurie-Vignal M. (2006), Droit de la distribution, Sirey Université, en particulier chapitre 2, section V, 107-124.

(9) Messeghem K. (2005), Les distributeurs en quête de légitimité : le cas des accords de coopération avec les PME, Décisions Marketing, 39, 57-66.

(10) Ministre des PME, du Commerce, de l'artisanat et des Professions libérales (2005), Point de presse du 6 septembre 2005, Mise en ouvre de la loi Galland, Evolution des prix des produits de grande consommation, et Projet de loi pour les PME, Transparence des pratiques commerciales, Ministère de l'économie et des finances.

(11) Mousseron M., Mousseron J.M., Mainguy D. (1998), Le droit français nouveau de la transparence tarifaire, Litec, Bibliothèque de droit de l'entreprise.

(12) Puget Y. (2006), Les PME confortent leur place dans les rayons, LSA, 1960, 6 juillet 2006, 20-21.

(13) Villain C. (1995), Rapport sur les relations entre l'industrie et la grande distribution. 
Encadré 1 : La grande distribution française, un oligopsone

Face aux multiples industriels, six centrales d'achat concentraient $90 \%$ des achats des biens de grande consommation, en France fin 2004 :

\begin{tabular}{|l|l|c|}
\hline \multicolumn{1}{|c|}{ Centrales d'achat } & \multicolumn{1}{|c|}{ Enseignes } & Parts de marché (\%) \\
\hline LUCIE & SYSTEME U, LECLERC & 25,7 \\
\hline CARREFOUR & $\begin{array}{l}\text { CARREFOUR, CHAMPION, SHOPI, MARCHE PLUS, } \\
\text { COMOD, HUIT A HUIT, ED }\end{array}$ & 23 \\
\hline AUCHAN & AUCHAN, ATAC & 13,1 \\
\hline EMC & $\begin{array}{l}\text { CASINO, GEANT CASINO, FRANPRIX, LEADER } \\
\text { PRICE, MONOPRIX }\end{array}$ & 12,6 \\
\hline ITM ENTREPRISES & $\begin{array}{l}\text { INTERMARCHE, ECOMARCHE, NETTO, } \\
\text { COMPTOIRS DES MOUSQUETAIRES }\end{array}$ & 11,9 \\
\hline PROVERA & CORA, MATCH & 3,7 \\
\hline
\end{tabular}

Source : Rapport Canivet (3)

Depuis, début 2006, SYSTEME U et LECLERC ont séparé leurs activités d'achat. SYSTEME U est entré dans EMD, European Marketing Distribution, la plus grosse centrale en Europe par le nombre d'adhérents et le chiffre d'affaires, et LECLERC a créé, avec 4 autres distributeurs étrangers une centrale d'achat européenne, COOPERNIC. 


\section{Tableau 1 - Les intérêts divergents des acteurs face à la loi Galland : les consommateurs ont été perdants, les distributeurs et multinationales ont été bénéficiaires. Le bilan est plus mitigé pour les PME.}

\begin{tabular}{|c|c|c|c|c|}
\hline & Consommateurs & Grands distributeurs & $\begin{array}{l}\text { Groupes industriels } \\
\text { (multinationales) }\end{array}$ & PME \\
\hline Objectifs & $\begin{array}{l}\text { Maintenir le pouvoir } \\
\text { d'achat, bénéficier de } \\
\text { baisse de prix } \\
\text { Bénéficier d'une offre } \\
\text { diversifiée }\end{array}$ & 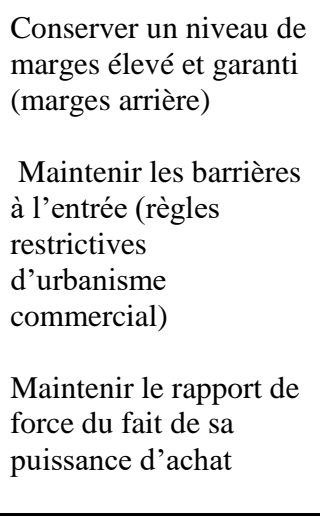 & $\begin{array}{l}\text { Conserver des marges } \\
\text { élevées et la maîtrise des } \\
\text { prix de vente au } \\
\text { consommateur } \\
\text { Renforcer la puissance } \\
\text { marketing/ } \\
\text { communication de ses } \\
\text { marques et l'attractivité } \\
\text { pour le consommateur } \\
\text { Maintenir son pouvoir de } \\
\text { négociation et sa place en } \\
\text { linéaires }\end{array}$ & $\begin{array}{l}\text { Exister sur les linéaires } \\
\text { malgré leur rareté } \\
\text { Conserver des volumes de } \\
\text { production } \\
\text { Maintenir des marques } \\
\text { propres (ne pas être réduit } \\
\text { à ne fabriquer que des } \\
\text { MDD, situation qui } \\
\text { accroît la dépendance vis } \\
\text { à vis des distributeurs) }\end{array}$ \\
\hline $\begin{array}{l}\text { Menaces du } \\
\text { fait de la } \\
\text { concentration de } \\
\text { la distribution } \\
\text { et de la } \\
\text { réglementation } \\
\text { (lois Galland et } \\
\text { Raffarin) }\end{array}$ & $\begin{array}{l}\text { Suppression de la } \\
\text { concurrence intra- } \\
\text { marque (entre } \\
\text { distributeurs, voir } \\
\text { glossaire en encadré 6) } \\
\text { résultat de la loi } \\
\text { Galland } \\
\text { Hausse des prix }\end{array}$ & $\begin{array}{l}\text { Développement du } \\
\text { hard-discount } \\
\text { Moins de } \\
\text { différenciation prix } \\
\text { (accent sur les services } \\
\text { et nouveaux } \\
\text { instruments } \\
\text { promotionnels) }\end{array}$ & $\begin{array}{l}\text { Développement des } \\
\text { marques de distributeurs } \\
\text { (MDD) }\end{array}$ & $\begin{array}{l}\text { Réduction des linéaires } \\
\text { pour marques propres } \\
\text { «challengers » } \\
\text { Développement des MDD } \\
\text { premier prix, pression sur } \\
\text { les prix (transparence des } \\
\text { marges pour les } \\
\text { distributeurs, concurrence } \\
\text { des PME étrangères...) }\end{array}$ \\
\hline $\begin{array}{l}\text { Solutions à court } \\
\text { terme }\end{array}$ & 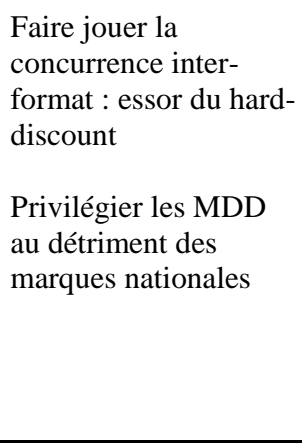 & $\begin{array}{l}\text { Maintenir/ augmenter } \\
\text { les marges arrières } \\
\text { Investir/ contrôler le } \\
\text { hard-discount } \\
\text { Créer des MDD } 1^{\mathrm{er}} \text { prix }\end{array}$ & $\begin{array}{l}\text { Renforcer les éléments de } \\
\text { différenciation } \\
\text { (innovation, puissance } \\
\text { marketing et de } \\
\text { communication) } \\
\text { Défendre ses positions } \\
\text { par des mesures telles que } \\
\text { les accords de gamme }\end{array}$ & $\begin{array}{l}\text { Innover et garder de la } \\
\text { réactivité pour imposer } \\
\text { les marques propres } \\
\text { «challengers » } \\
\text { Eviter la pression à la } \\
\text { baisse des marges sur } \\
\text { MDD et/ou réaliser des } \\
\text { efforts de productivité } \\
\text { Construire la confiance } \\
\text { sur long terme }\end{array}$ \\
\hline $\begin{array}{l}\text { Positions par } \\
\text { rapport à la } \\
\text { réglementation }\end{array}$ & $\begin{array}{l}\rightarrow \text { remise en cause loi } \\
\text { Galland }\end{array}$ & $\rightarrow$ statu quo & $\begin{array}{l}\rightarrow \text { statu quo (cercle } \\
\text { vertueux) }\end{array}$ & $\begin{array}{l}\rightarrow \text { Bilan mitigé } \\
\text { volumes de production au } \\
\text { prix d'une dépendance } \\
\text { accrue }\end{array}$ \\
\hline
\end{tabular}


Tableau 2 - Les définitions successives du seuil de revente à perte (SRP) et leurs effets potentiels sur le prix de vente au consommateur.

\begin{tabular}{|c|c|c|c|c|}
\hline & $\begin{array}{c}\begin{array}{c}\text { Avant la loi Galland } \\
\text { (ordonnance Balladur } \\
\text { 1986) }\end{array} \\
\end{array}$ & $\begin{array}{l}\text { SRP loi Galland } \\
1996\end{array}$ & SRP Dutreil 2006 & SRP Dutreil 2007 \\
\hline $\begin{array}{l}\text { Définition SRP } \\
=\text { Prix de vente } \\
\text { minimum }\end{array}$ & $\begin{array}{l}\text { Prix d'achat unitaire } \\
\text { - remises, rabais et } \\
\text { ristournes (RRR) } \\
\text { attachées directement } \\
\text { à l'opération ou non }\end{array}$ & $\begin{array}{l}\text { Prix d'achat unitaire } \\
\text { - réductions } \\
\text { directement attachées } \\
\text { à l'opération d'achat } \\
\text { et acquises à la date } \\
\text { de la vente } \\
\text { + taxes } \\
\text { + prix du transport }\end{array}$ & $\begin{array}{l}\text { SRP Galland } \\
- \text { le plus petit des } \\
\text { deux chiffres } \\
\text { [marges arrière }-20 ; \\
40 \% \text { des marges } \\
\text { arrière] }\end{array}$ & $\begin{array}{l}\text { SRP Galland } \\
-(\text { marges arrière }-15)\end{array}$ \\
\hline $\begin{array}{l}\text { Prix de vente } \\
\text { consommateur } \\
\text { minimum dans } \\
\text { l'exemple }\end{array}$ & $\begin{array}{l}\text { Très inférieur à } 100 \\
\text { si imputation sur une } \\
\text { facture de RRR } \\
\text { accumulés (pratique } \\
\text { de la cagnotte) }\end{array}$ & 100 & $\begin{array}{c}86 \\
100-(\text { plus petit }[35 \\
-20 ; 40 \% \times 35])\end{array}$ & $\begin{array}{c}80 \\
100-(35-15)\end{array}$ \\
\hline $\begin{array}{l}\text { Marges possibles } \\
\text { du distributeur si } \\
\mathrm{PV}=100\end{array}$ & $\begin{array}{l}\text { Marges négatives } \\
\text { possibles (prix } \\
\text { d'appel) }\end{array}$ & $\begin{array}{l}\text { Marges avant }=0 \\
\text { Marges arrière }=35\end{array}$ & $\begin{array}{l}\text { Marges avant }=14 \\
\text { Marges arrière }=20\end{array}$ & $\begin{array}{l}\text { Marges avant }=20 \\
\text { Marges arrière }=15\end{array}$ \\
\hline $\begin{array}{l}\text { Décisions } \\
\text { possibles du } \\
\text { distributeur }\end{array}$ & $\begin{array}{l}\text { Ilot de pertes dans un } \\
\text { océan de profit }\end{array}$ & $\begin{array}{l}\text { Gonfler les marges } \\
\text { arrière pour accroître } \\
\text { sa rémunération }\end{array}$ & $\begin{array}{l}\text { Se différencier des } \\
\text { concurrents par le } \\
\text { prix de vente : } \\
\text { renoncer aux marges } \\
\text { avant }\end{array}$ & $\begin{array}{l}\text { Se différencier des } \\
\text { concurrents par le } \\
\text { prix de vente : } \\
\text { renoncer aux marges } \\
\text { avant }\end{array}$ \\
\hline Dérives possibles & & & \multicolumn{2}{|c|}{$\begin{array}{l}\text { Partage imposé de la baisse des marges } \\
\text { avant par les industriels } \\
\text { Création de nouvelles sources de marges, } \\
\text { pour maintenir le niveau de profit } \\
\text { Hausse des tarifs des industriels à fort } \\
\text { pouvoir de négociation (voir encadré 5) }\end{array}$} \\
\hline
\end{tabular}




\section{Encadré 2 : Condamnation par le Tribunal de commerce de Nanterre de LECLERC à rembourser 23,3 millions d'euros indûment perçu au titre de la coopération commerciale, mais ... infirmée par la Cour d'appel de Versailles}

Apprenant, en septembre 2001, que ses fournisseurs consentaient à CARREFOUR, pour des produits alimentaires frais, des conditions de coopération commerciale, c'est à dire des marges arrière, plus avantageuses que celles qui lui étaient accordées, la centrale de référencement de LECLERC, Le Galec, en charge des négociations avec les fournisseurs, estime qu'il y a discrimination, et demande réparation à ces derniers, c'est à dire le remboursement de 23,3 millions d'euros. Les 23 fournisseurs acceptent de signer, rétroactivement, des contrats de coopération commerciale pour les années 98 et 99, et paient.

Saisi par la DGCCRF, et non par les fournisseurs concernés, le tribunal de commerce de Nanterre condamnait LECLERC à rembourser aux industriels les 23,3 millions soutirés et à payer une amende civile de 500000 euros (jugement du 15 novembre 2005). Les motifs de la décision étaient que les accords rétroactifs sont contraires à l'article L 442-6 II a) du code de commerce qui énonce « Sont nuls les clauses ou contrats prévoyant pour un distributeur la possibilité de bénéficier rétroactivement de remises, de ristournes ou d'accords de coopération commerciale. » Mais le tribunal ajoutait que les contrats ne correspondaient à aucun service commercial effectivement rendu, rappelant l'exigence de services justifiés par une contrepartie réelle et non disproportionnée.

Le jugement était remarqué tant pour les montants en jeu, que pour les modalités pratiques : craignant que les industriels, par peur des représailles, ne réclament les sommes litigieuses à LECLERC (comment réclamer en effet ces sommes à un client qui représente quelques $20 \%$ de son CA potentiel, et avec lequel sont négociés les budgets 2006 ?), le tribunal prévoyait que les 23,3 millions seraient restitués par l'intermédiaire du Trésor Public, qui recouvrerait les sommes et les verserait ensuite aux industriels...c'était astucieux, mais sans doute trop : la Cour d'appel de Versailles, saisie par LECLERC, a infirmé la décision du tribunal, dans un arrêt du 3 mai 2007, soulignant un vice de procédure : le ministre de l'économie ne pouvait introduire l'action en justice sans informer et associer les industriels. Or 17 d'entre eux avaient expressément exprimé leur volonté contraire. L'affaire est portée devant la Cour de cassation.

Sources : Tribunal de commerce de Nanterre, jugement prononcé le 15 novembre 2005, $7^{\text {ème }}$ chambre, affaire 2004F01493, 6 pages ; Cour d'appel de Versailles, arrêt nº 05.09223 du 3 mai 2007. 


\section{Tableau 3 - l'encadrement des allocations de budgets des industriels aux distributeurs}

\begin{tabular}{|c|c|c|}
\hline Budgets alloués & Définitions & Exemples de contenu \\
\hline $\begin{array}{l}\text { Conditions } \\
\text { Générales de } \\
\text { vente }(\mathrm{CGV})\end{array}$ & $\begin{array}{l}\text { Prix tarifs fixés par les industriels et remises consenties sur } \\
\text { la même base à tous les distributeurs ; point de départ des } \\
\text { négociations ; elles doivent être communiquées à tout client } \\
\text { potentiel ; } \\
\text { Possibilités de CGV catégorielles (selon les types d'acteurs : } \\
\text { centrales, magasins...), qui restent à confirmer par décret }\end{array}$ & $\begin{array}{l}\text { Tarifs, remises qualitatives et } \\
\text { quantitatives telles que les } \\
\text { remises de gamme, les } \\
\text { ristournes conditionnelles non } \\
\text { acquises au moment de l'achat } \\
\text { (ristournes de fin d'années, ou } \\
\text { remises d'objectifs, fonction } \\
\text { du volume d'achats) }\end{array}$ \\
\hline $\begin{array}{l}\text { Contrat de } \\
\text { coopération } \\
\text { commerciale }\end{array}$ & 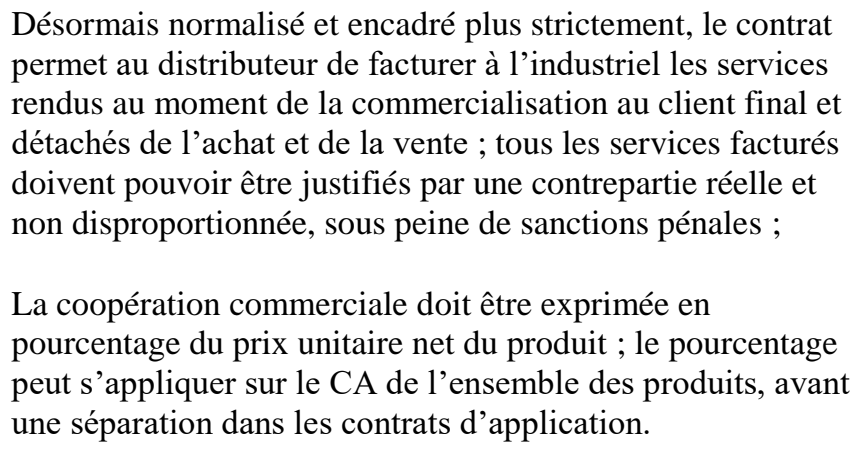 & $\begin{array}{l}\text { Têtes de gondole, } \\
\text { Mises en avant, } \\
\text { Diffusion de prospectus et } \\
\text { publi-promotions, tracts... } \\
\text { Gestion des coupons et autres } \\
\text { nouveaux instruments } \\
\text { promotionnels (NIP), conçus } \\
\text { pour contourner l'interdiction } \\
\text { de vente à perte }\end{array}$ \\
\hline $\begin{array}{l}\text { Conditions } \\
\text { particulières de } \\
\text { vente }(\mathrm{CPV})\end{array}$ & $\begin{array}{l}\text { Elles ouvrent la voie à la pratique de différenciation } \\
\text { tarifaire ; Elles doivent être fondées sur des services } \\
\text { spécifiques rendus par l'acheteur (le distributeur). } \\
\text { Elles peuvent être secrètes (pas d'obligation d'information) } \\
\text { elles constituent une discrimination licites (8). Elles } \\
\text { pourraient constituer un réservoir de marges bénéficiaires, } \\
\text { comme la coopération commerciale. }\end{array}$ & $\begin{array}{l}\text { Prestations logistiques à } \\
\text { l'occasion de la livraison des } \\
\text { produits, ou un service de } \\
\text { stockage particulier }\end{array}$ \\
\hline $\begin{array}{l}\text { Services } \\
\text { distincts }\end{array}$ & $\begin{array}{l}\text { Services facturés autres que ceux relevant de la coopération } \\
\text { commerciale ; la liste n'est pas exhaustive, sert de « voiture } \\
\text { balai »: inclut les budgets facturés qui ne le sont pas ailleurs } \\
\text { et qui ne visent pas le consommateur. } \\
\text { La forme est moins encadrée, mais la réalité des services } \\
\text { rendus est soumise aux mêmes contrôles que pour les } \\
\text { services de coopération commerciale. Et le juge pourra } \\
\text { contrôler qu'ils ne relèvent pas de la coopération } \\
\text { commerciale. }\end{array}$ & $\begin{array}{l}\text { Ventes de statistiques et autres } \\
\text { données « sorties des caisses », } \\
\text { Action de formation du } \\
\text { personnel, } \\
\text { Services de collaboration } \\
\text { internationale ou autres } \\
\text { services de nature inter- } \\
\text { industrielle }\end{array}$ \\
\hline
\end{tabular}


Encadré 3 : Techniques juridiques pour rendre effective l’interdiction de « fausse coopération »

En droit français, la preuve incombe au demandeur, c'est-à-dire à celui qui veut faire reconnaître son droit (Article 1315 du code civil). Toutefois, les textes peuvent prévoir, dans certains cas, que la preuve doit être apportée par la partie adverse, lorsque la preuve est difficile à établir pour le demandeur ou lorsqu'il faut protéger une partie «faible ». On parle alors de « renversement de la charge de la preuve ».

Dans les rapports entre distributeurs et industriels, les règles normales de la charge de la preuve obligeraient l'industriel à apporter la preuve qu'une facturation, au titre de la coopération commerciale ou au titre de services distincts, est abusive car elle ne correspond à aucun service réel rendu par le distributeur. Mais on voit mal les industriels, qui dépendent de la grande distribution pour avoir accès au consommateur, ester en justice pour faire condamner les distributeurs. C'est pourquoi la circulaire Dutreil prévoit :

- l'auto-saisine de la DGCCRF, Direction Générale de la Concurrence, de la Consommation, et de la Répression des Fraudes, dotée de pouvoirs d'investigation étendus, même en l'absence d'action ou de réclamation d'un industriel

- le renversement de la charge de la preuve : c'est au distributeur qui a obtenu une réduction de prix au titre des $\mathrm{CGV}$ ou des $\mathrm{CPV}$, d'apporter la preuve que cette réduction rémunère un service réel et non disproportionné.

Les nouveaux textes redonnent de la liberté aux distributeurs, mais les exposent à un risque pénal accru.

La loi prévoit, en cas d'infraction aux nouvelles règles, outre une inscription au casier judiciaire, des amendes allant jusqu'à 75000 euros pour les personnes physiques et jusqu'à 375000 euros pour les personnes morales.

A souligner aussi : la DGCCRF multiplie les contrôles en matière de relation commerciale, $+9,3 \%$ en 2005, et dispose, depuis un décret du 4 mai 2006, d'un pouvoir de transaction en cas d'infraction. Elle peut désormais proposer, sous contrôle du procureur de la République, une transaction à l'auteur d'une infraction, et fixer le montant de l'amende. Ce dernier aura un mois pour accepter et éviter ainsi l'action devant les tribunaux. 


\section{Encadré 4 : Essor du hard-discount et contre-offensive de la grande distribution classique.}

Eléments chiffrés concernant le hard-discount :

- $\quad$ il représente 13, $4 \%$ de part de marché valeur en 2005, soit une progression de 4, $3 \%$ en 5 ans ;

- $\quad$ avec un net ralentissement en 2005 par rapport à 2004 : la hausse n'est plus que de 0,1 sur un an ;

- $\quad$ il compte 3700 points de vente en 2005, soit 1000 de plus qu'en 2001

6 enseignes sont présentes en France en 2005 :

- deux sont d'origine allemande : LIDL (numéro 1 en France, avec 1200 points de vente, et plus de $30 \%$ de PDM du hard discount) et ALDI (705 points de vente, $16 \%$ de PDM du hard-discount)

- les autres appartiennent aux distributeurs traditionnels français: LEADER PRICE (groupe CASINO) avec $26 \%$ de PDM, ED (groupe CARREFOUR) avec $17 \%$ de PDM, NETTO (groupe INTERMARCHE) et LE MUTANT (LES COOPERATIVES DE NORMANDIE) se partagent le reste.

La contre- offensive de la distribution classique s'est traduite par :

- $\quad$ une offre renforcée de MDD et produits premier prix : pour la première fois en avril 2006, leur part de marché en volume dépasse celle des marques nationales, dans les formats classiques ;

- une concentration de l'offre de marques nationales sur les références les plus performantes ;

- la création de « coins discount » dans les formats traditionnels ;

- le lancement de nouveaux concepts discount par les enseignes de distribution classiques. Par exemple, ATAC du groupe AUCHAN, absent jusqu'alors sur le format hard-discount teste un concept «Simply Market» à Bagneux, formule entre le supermarché qualitatif et le discount, CASINO a lancé des points de vente «Eco Discount » et «Géant Discount ».

Sources : Etude annuelle TNS Secodip, IRI France, Que Choisir (5). 


\section{Encadré 5 : Atténuer les effets de la loi Dutreil-Jacob par une hausse significative des tarifs des industriels à fort pouvoir de négociation. Exemple.}

Reprenons l'exemple développé dans le tableau 2, c'est à dire celui d'un produit dont le prix achat tarif est de 110, les remise sur factures de 10, ce qui donne un SRP de 100, et des marges arrière de 35. Imaginons que l'industriel opère une hausse du tarif net, à 115, et propose une hausse des marges arrière de 10 points, soit 45 .

Le SRP devient : 100 - plus petit [45-20; $40 \%$ x 45], soit 97. La baisse de prix effective au consommateur sera de $3 \%$ maximum, l'industriel a augmenté ses marges (qui compenseront plus que largement la hausse des coûts de l'énergie). La rémunération du distributeur, avant négociation, sera comprise entre 30 et 27 , en fonction de sa politique commerciale : elle sera de 30, s'il laisse le prix consommateur à 100 et prend une marge avant de 3 (rémunération $=100-115+45=3$ ), et de 27 s'il opère une baisse de prix. Contre une rémunération de 35 avant la réforme.

Si les marges arrière sont portées à 50, le SRP sera de 95, et la marge du distributeur comprise entre 32 et 35. Mais encore faut-il pouvoir justifier la réalité des services facturés 50, alors qu'ils étaient facturés 35 avant la réforme ! le risque juridique, pénal, supporté par le distributeur est une limite à cette riposte.

Les observateurs annoncent des hausses de tarifs sur les marques « incontournables », celles de L'OREAL, UNILEVER, DANONE, PROCTER et GAMBLE etc., de plus de 5 à 10 points lors des négociations 2006 (1). 


\section{Encadré 6 : Glossaire}

Accords de gamme : accords qui offrent un avantage, financier ou autre, en contrepartie de la mise en linéaire d'une gamme de produits. Ils peuvent conduire à entraver l'accès de produits similaires aux points de vente, notamment les produits des PME, « outsiders ». La loi Dutreil entend sanctionner leur caractère abusif (apprécié par le juge).

Concurrence intra-marque : guerre des prix que se livrent les distributeurs/enseignes sur un produit de marque fourni par les industriels. Par exemple, concurrence prix entre un hyper CARREFOUR et un hyper AUCHAN et un super LECLERC d'une même zone de chalandise, sur un produit phare tel que les yaourts aux fruits de la marque DANONE. La concurrence intra-marque peut être intra-format ou inter-formats.

Concurrence intra-format (ou intra-type) : concurrence prix entre points de vente pratiquant la même forme de vente. Par exemple, guerre des prix entre un hyper AUCHAN et un hyper CARREFOUR d'une même zone de chalandise, sur les yaourts aux fruits de la marque DANONE.

Concurrence inter-formats (ou inter-types) : concurrence prix opposant des formes de ventes différentes. Par exemple, concurrence prix entre un supermarché de proximité et un magasin hard-discount sur la même zone de chalandise, sur les yaourts aux fruits de la marque DANONE.

CGV ou conditions générales de vente : vivement recommandées, elles sont établies par le fournisseur. Elles précisent les modalités de vente offertes à ses clients, elles comprennent généralement les barèmes de prix, réductions de prix à caractère quantitatif ou qualitatif, les conditions de règlement. Elles doivent être communiquées à tout acheteur qui en fait la demande pour son activité professionnelle.

CPV ou conditions particulières de vente : la loi du 2 août 2005 précise que les CGV ne font pas obstacle à ce qu'une différenciation tarifaire des clients soit réalisée. Des CPV peuvent être négociées pour tenir compte de la spécificités des services rendus, tels que les services logistiques à l'occasion de la livraison des produits, ou un service de stockage différencié. Elles doivent être justifiées par une contrepartie.

Discrimination tarifaire : c'est le fait de traiter différemment des clients présentant les mêmes caractéristiques et ayant les mêmes demandes. Notion créée par la loi Galland, et précisée par la loi du 2 août 2005 - à distinguer de la différenciation tarifaire, légale en cas de conditions différenciées, justifiées par une contrepartie, par exemple des modalités de livraison, stockage, services logistiques, engagements d'achat prédéterminés, modalités de règlement... ou si elle est catégorielle c'est à dire si elle prévoit des conditions distincts selon les catégories d'acheteurs, notamment grossistes et détaillants (un décret, à paraître doit préciser ces catégories).

Hard-discount : libre service alimentaire avec un personnel réduit, une présentation des produits sommaire, un assortiment limité aux produits de base, des prix bas, peu de marques nationales (en hausse toutefois), mais des marques de distributeurs ou des produits sans marques.

Marges arrière : remises hors facture comme les réductions de prix sujettes à la réalisation d'une condition (remises d'objectifs) et la rémunération de services proposés à l'industriel au titre de la coopération commerciale (voir cidessous). La dénomination de marge est abusive car il ne s'agit pas d'un différentiel entre un prix de vente et un prix d'achat (ce différentiel étant désormais appelé marges avant); Les marges arrière constituent une rémunération garantie pour le distributeur, qui permet de l'analyser comme un «loueur d'espace» plus que comme un commerçant. Les marges arrière ont connu des hausses très importantes après la loi Galland.

Oligopsone : en économie, marché sur lequel il y a un petit nombre d'acheteurs pour un grand nombre de vendeurs. La grande distribution française compte moins de 10 groupes de distribution acheteurs face aux multiples industriels.

SRP ou seuil de revente à perte : prix plancher, en dessous duquel le distributeur ne peut pas revendre les produits, sauf à enfreindre l'interdiction de revente à perte et à s'exposer à des sanctions pénales lourdes. Sa définition a varié dans le temps, voir tableau 2.

Services de coopération commerciale : actions de nature à stimuler au bénéfice du fournisseur (industriel) la revente de ses produits au consommateur par le distributeur, par exemple les opérations publi-promotionnelles, la publicité sur les lieux de vente, l'attribution de têtes de gondoles, etc. Cette définition stricte résulte de la loi DutreilJacob qui précise que les services de coopération commerciale répondent à trois caractéristiques : ils ne relèvent pas des obligations d'achat et de vente, ils sont rendus à l'occasion de la revente des produits ou des services aux consommateurs, ils sont de nature à favoriser la commercialisation des produits ou services.

Services distincts : ceux qui ne remplissent pas les conditions des « services de coopération commerciale », par exemple les ventes de statistiques «sorties des caisses », ou les services offerts par le distributeur dans le cadre d'accords internationaux négociés en dehors du territoire national, ou les services rendus par un grossiste à son fournisseur, qui ne le sont pas à l'occasion de la revente des produits aux consommateurs. 